University of the Pacific Scholarly Commons

$7-1-2018$

\title{
Time out for sibling aggression: An analysis of effective durations in a natural setting
}

Samantha M. Corralejo

University of the Pacific

Scott A. Jensen

University of the Pacific, sjensen@pacific.edu

Ashley Dawn Greathouse

University of South Alabama

Follow this and additional works at: https://scholarlycommons.pacific.edu/cop-facarticles

Part of the Psychology Commons

\section{Recommended Citation}

Corralejo, S. M., Jensen, S. A., \& Greathouse, A. D. (2018). Time out for sibling aggression: An analysis of effective durations in a natural setting. Child and Family Behavior Therapy, 40(3), 187-203. DOI: 10.1080/07317107.2018.1487701

https://scholarlycommons.pacific.edu/cop-facarticles/610 
Running head: TIME-OUT ANALYSIS OF DURATIONS

Time-out for Sibling Aggression: An Analysis of Effective Durations in a Natural Setting Samantha M. Corralejo, Scott A. Jensen, and Ashley Dawn Mitchell University of the Pacific

Author Note

Samantha M. Corralejo, Department of Psychology, University of the Pacific, samantha.corralejo@aggiemail.usu.edu; Scott A. Jensen, Department of Psychology, University of the Pacific, sjensen@pacific.edu; Ashley Dawn Mitchell, Department of Psychology, University of the Pacific, am1629@jagmail.southalabama.edu.

Samantha M. Corralejo is now at Department of Psychology, Utah State University. Ashley Dawn Mitchell is now at Department of Psychology, University of South Alabama. Correspondence concerning this article should be addressed to Samantha M. Corralejo, Department of Psychology, Utah State University, 2810 Old Main Hill, Logan, UT 84322.

E-mail: samantha.corralejo@aggiemail.usu.edu

Phone: (805) 279-1863

Abstract 
TIME-OUT ANALYSIS OF DURATIONS

Time-out is a ubiquitous intervention strategy to reduce problem behaviors. The current study sought to find the shortest effective duration(s) of time-out necessary to reduce sibling aggression in a community sample of girls ages 3-7. The intervention took place in participants' homes using a minute-by-minute incremental increase and reversal design. All participants reached a minimum reduction in sibling aggression of $60 \%$ after experiencing a 1-min time-out. The majority (75\%) of participants also demonstrated clear reversals of behavior when returned to the baseline condition. The current findings suggest that a 1-min time-out may be sufficient for low-level sibling aggression in children as old as seven. Limitations include the presence of a graduate assistant during sibling play and some loss of experimental control in the natural setting. Future research should seek to replicate the current methodology with the same population and populations of different ages and developmental levels. Keywords: time-out, sibling aggression, duration, behavior modification

Time-out for Sibling Aggression: An Analysis of Effective Durations in a Natural Setting Research has repeatedly demonstrated the effectiveness of time-out as a behavior 
TIME-OUT ANALYSIS OF DURATIONS

reduction technique in children (Baer, 1961; Bostow \& Bailey, 1969; Burchard \& Barrera, 1972;

James, 1976; Kendall, Nay, \& Jeffers, 1975; Wahler, 1969; White, Nielsen, \& Johnson, 1972).

Time-out is a form of negative punishment generically defined as "a period of time in which positive reinforcement is no longer available" (Leitenberg, 1965, p. 428). Early research found time-out to be effective in reducing problem behaviors in children with developmental disabilities (Bostow \& Bailey, 1969). Subsequent research demonstrated its usefulness in reducing undesirable behaviors, varying from non-compliance, to aggressive behaviors, to stuttering (Burchard \& Barrera, 1972; Donaldson \& Vollmer, 2011; Fabiano et al., 2004; James, 1976; Kapalka \& Bryk, 2007; Kendall et al., 1975; MacDonough \& Forehand, 1973; Wahler, 1969; White et al., 1972).

MacDonough and Forehand (1973) provided a useful structure for understanding the various aspects of time-out by outlining eight parameters of time-out: location/supervision, schedule, warning, signal, form of administration, verbalized reason, contingent release, and duration. Duration is perhaps the most studied of these parameters, and yet ongoing debate as to the most effective durations continues. Duration is a key parameter because of the ethical implications of excessively long time-outs, the popular yet empirically unsupported belief that duration should increase with age, and the variable research findings on effective durations thus far (Fabiano et al., 2004; White et al., 1972).

Studies on duration have commonly assessed lengths between 1 minute and 1 hour. Interestingly, one of the most consistent findings is the presence of sequence effects when multiple time out durations are used within the same subjects. In general, researchers have found that when a longer duration time-out is followed by a lesser duration time-out, problem behaviors increase (Burchard \& Barrera, 1972; Kendall et al., 1975; White et al., 1972). Beyond 
TIME-OUT ANALYSIS OF DURATIONS

sequence effects, durations 15 minutes and longer tend to be equally effective in reducing problem behavior (Benjamin, Mazzarins, \& Kupfersmid, 1983; White et al., 1972). Because of this, researchers often discourage the use of unnecessarily long time-outs. Findings for time-out durations less than 15 minutes have been more variable, with some studies supporting durations as short as 1 to 4 minutes (Fabiano et al., 2004; McGuffin, 1991). Shorter durations have not been found to be more effective than longer durations, only equally effective. Hobbs, Forehand, and Murray (1978) focused on analyzing time-out durations less than 5 minutes with mothers and their children. Ten second, 1-min, and 4-min time-outs all reduced deviant behavior, however 4-min resulted in the greatest, most maintained reductions (Hobbs et al., 1978).

The notion that the duration of time-out should increase as age increases ( 1 to 2 minutes per year of age) is commonly seen in popular parenting sources (Clark, 2005; Frost, 2014; Reece, 2013; Smith \& Chandler, 2010; Zolten \& Long, 1997). In a sample of 4- and 5-year-old children with ADHD, Kapalka and Bryk (2007) compared set lengths of time out (2- and 4-min) to timeout durations based on the child's age (1- and 2-mins for each year) and found no difference in outcome based on age

Unfortunately, the majority of time-out research is conducted in clinical settings, with therapist or other trained individuals implementing time-out, with relatively little research including parent use of time-out (see Corralejo, Jensen, Greathouse, and Ward, 2017 for a more detailed review of the time-out and duration literature). One common source of externalizing behavior problems is sibling conflict. Home observations have revealed that conflict occurs during up to $29 \%$ of interactions and parents have reported aggression and violence from one child to another as common issues (Dunn, 1983; Kramer \& Baron, 1995; Straus, Gelles, \& Steinmetz, 1981). Time-out has repeatedly demonstrated success in reducing sibling aggression 
TIME-OUT ANALYSIS OF DURATIONS

in children as young as one and as old as 12 (Adams \& Kelley, 1992; Allison \& Allison, 1971;

Jones, Sloane, \& Roberts, 1992; O’Leary, O’Leary, \& Becker, 1967; Olson \& Roberts, 1987).

The present study sought to identify the shortest effective duration(s) of time-out for a community sample of sibling dyads. We sought to improve upon two key aspects of previous research. First, whereas most of the previous research used trained implementers (therapists), occurred in an unnatural or contrived clinical setting, or relied on parent reported outcomes (e.g., Hobbs \& Forehand, 1975; Hobbs et al., 1978), the current research collected in-vivo observational data and ensured treatment integrity while the parent implemented the intervention in their home environment. Second, whereas duration research typically assesses two to four pre-specified durations, often encountering problems with sequence effects (Burchard \& Barrera, 1972; Fabiano et al., 2004; Hobbs et al., 1978; McGuffin, 1991; Pendergrass, 1971), the present study started with a 1-min time-out and planned to increase the duration incrementally until timeout was effective (a minute-by-minute incremental increase). Barton, Brulle, and Repp (1987) used a similar "increase until effective" design and found that they only needed to increase the duration for one of three children to reach targeted suppression levels. Because research has identified time-out as an appropriate intervention for sibling aggression, we deemed sibling aggression an appropriate and ecologically valid target behavior for the current study (Adams \& Kelley, 1992; Allison \& Allison, 1971; Jones et al., 1992; O’Leary et al., 1967; Olson \& Roberts, 1987).

\section{Method}

\section{Participants}

We recruited a total of six families through referrals from community organizations such 
TIME-OUT ANALYSIS OF DURATIONS

as childcare services and abuse prevention organizations and we collected data on the four families that qualified for participation in our study. Two families did not meet eligibility criteria after the children engaged in low baseline rates of aggression. We collected data for each sibling to track individual responses to the intervention. Participants ranged in age from 3-7 (see Table 1). Criteria for participating in the study were as follows: the family included two siblings between the ages of 3 and 8 and the parent reported sibling play-related conflict and aggression as occurring a minimum of once a day, five days a week (on average, tracked by the parent using a behavior tracking sheet). Parents had been using time-out inconsistently $(n=3)$ or not at all $(n=1)$; if a parent had been consistently using time-out to target a specific behavior they would have been excluded from the study. Conflict and aggression were defined as a physical interaction “...involving one or both of the siblings that include[s] hitting, pushing, kicking, spitting, biting, throwing objects, or struggling over toys" (as per Olson \& Roberts, 1987, p. 245). Each child counted as a participant and received her own intervention.

All siblings were female as was the primary caretaker implementing the intervention. Pseudonyms are used in place of the children's actual names. Two children had been diagnosed with ADHD (Lisa and Dakota); Dakota also had been diagnosed with static encephalopathy (permanent brain damage). Ashley had been diagnosed with Noonan Syndrome, a condition primarily affecting physical development. The remaining five children were typically developing, making the participants included a true community sample with a mixture of typically developing and non-typically developing children. The participants were ethnically diverse.

\section{Design, Setting, Materials, and Procedure}

We employed a within-subject reversal design to identify an effective intervention and 
TIME-OUT ANALYSIS OF DURATIONS

assess its reversibility. Once a specific duration of time-out suppressed the target behavior to two instances or fewer in a given session, the reversal began.

The study consisted of a one-week preliminary tracking period, one main in-home meeting and a one-week post tracking period. Parents were to refrain from using time-out during the initial one-week tracking period as an attempt to prevent sequence effects. Because parents were not using time-out consistently to target a specific behavior, we did not see asking parents to refrain from using time-out as problematic. The in-home meeting included time-out training immediately followed by the intervention; this meeting lasted approximately $2.5 \mathrm{hrs,} \mathrm{depending}$ upon the number of treatment sessions required.

Initial training. We used a role-play practice and feedback teaching method developed by Marcus, Swanson, and Vollmer (2001) and demonstrated effective for training parents on a time-out procedure by Jensen, Blumberg, and Doerr (2017). Task analysis steps included choosing a non-reinforcing location, keeping the child in time-out for the correct amount of time, ignoring the child during time-out, and following through appropriately when time-out was over (see Appendix for full task analysis). After an initial discussion of the steps for time-out, participants practiced repeatedly while receiving initially immediate (within role-play) feedback, followed later by post-role-play feedback until they were able to implement time-out correctly without feedback twice in a row. All four parents reached $100 \%$ integrity on the time-out procedure through the brief training and maintained integrity throughout the in-home intervention. Data were not collected for parent integrity during follow-up, however all but one parent confirmed that they implemented time-out for every instance of sibling aggression tracked. While the parent trained on the time-out procedure, a graduate assistant conducted informal preference assessments while playing with the participants. The purpose of the time 
TIME-OUT ANALYSIS OF DURATIONS

spent with the children was threefold: to identify toys that might be reinforcing for both children (e.g., any toys that belonged to one child that the child was reluctant to share, a novel toy for singular child play brought by researchers that both children expressed interest in playing with), to gain understanding of the sibling dyad in terms of when conflict was most likely to arise (e.g., which sibling initiates aggression, which sibling finds sharing to be harder), and to explain the upcoming intervention and its purpose to the siblings (i.e., to help them get along better).

Baseline I. We recorded frequency of sibling aggression in two to three 5-min baselines. A rule was set at the beginning of the intervention that the participants could only play with one toy at a time, with the option of changing toys when they grew tired of one. The graduate assistant played with the children throughout the intervention to enforce this rule and encourage joint play with one toy. This is one way in which our 2-3 hour procedure was somewhat contrived; in the natural environment children often have multiple toys available to them at a time and do not have an adult prompting them to play continuously. For all sessions the parent could provide brief attention to the children (e.g., allowing their child to show them a toy, giving a hug) if aggression was not occurring; parent/child interactions that interrupted sibling play resulted in discontinuing data recording for that time. When aggression was occurring we only intervened if competition for a toy escalated to hitting, biting, and/or scratching, in which case we separated the children and redirected them to other activities until they no longer mentioned the toy they were previously fighting over (ie., this was only required once during data collection). To be eligible for intervention the child needed to engage in aggressive behavior for an average of five consecutive or nonconsecutive 5-sec intervals per 5-min measurement (a rate of one occurrence per minute) for two or more of the baseline measurements.

Treatment I. Participants that met inclusion criteria began treatment with a play 
TIME-OUT ANALYSIS OF DURATIONS

condition with 1-min time-outs contingent on sibling aggression. At the beginning of Treatment (and the second implementation of this condition, called Treatment II) we explained to the child that time-out would be a consequence for aggression. Children were told what each condition was going to be in order to provide quick understanding of each condition's consequences. Each condition consisted of sessions lasting 5 minutes each, excluding time spent in time-out. The number of sessions in Treatment I ranged from 1-3, depending upon exposure and reaction to the time-out contingency. For example, Tia and Tenley had two 1-min time-out sessions because in the first session neither participant aggressed and thus never contacted the contingency (see Figure 1). We ensured time-out was a consequence for every instance of aggression by signaling to the parent to implement time-out if the parent did not respond within 3 seconds. If the child engaged in the problem behavior for two or fewer 5-sec intervals, the treatment session discontinued, and the intervention progressed to Baseline II. If, after contacting the contingency, the child engaged in the problem behavior for more than two 5-sec intervals, the next session of training would have begun, which would have consisted of a 2-min increase in time-out length. This 1-min session increase would have continued as long as the child exhibited the target behavior above the cutoff criterion, or until a 10-min time-out was reached (no participant required an increase in duration - hence the hypotheticals).

Baseline II. No consequence occurred for aggression during the second 5-min baseline(s), with the same exception for hitting, biting, and/or scratching as in Baseline I. Before beginning we notified children that time-out was no longer going to occur for a while. If child behavior did not increase (in comparison to the final Treatment I session) during the first Baseline II session, a second Baseline II session was added. Similar to Treatment I sessions, an additional session during Baseline II allowed for adequate exposure to the new contingencies in 
TIME-OUT ANALYSIS OF DURATIONS

place.

Treatment II. Immediately following Baseline II a second treatment condition occurred. The duration of time-out was the last effective duration from Treatment I (1-min for all participants). We continued to observe play and gave the direction to implement time-out if needed. If frequency of aggression increased during Baseline II and did not decrease in the first session of Treatment II, a second 5-min session was added.

Debrief. At the end of Treatment II we explained to the parent how to teach appropriate replacement behaviors for sibling conflict and began the teaching process with the participants. This teaching included discussing problem-solving skills with the children, teaching the skill of sharing, and reinforcing instances of these behaviors when they occurred.

Data Analysis. We used visual analysis to examine the usefulness of specific durations of time-out. Since inclusion required Baseline I frequencies to be at five intervals per session or higher and an effective time-out duration was defined as two occurrences per session or less, there was a minimum of a $60 \%$ decrease in sibling aggression.

\section{Procedural Fidelity and Interobserver Agreement}

To ensure parent fidelity to the time-out procedure, we used session recordings to code $33 \%$ of all time-outs given. Video of time-out implementation was recorded for six of the eight participants (three families). Therefore, we coded $40-45 \%$ of the time-outs for three of the families. When coding we counted simultaneous sibling time-outs as one time-out to avoid redundancy. We coded each time-out implementation for the number of steps correctly completed out of 10 steps (see Appendix). We calculated fidelity for each implementation by dividing the total number of correct steps by the total number of steps. Parent fidelity to the time out procedure was high overall: across the three families with video to code there was $95.8 \%$ 
TIME-OUT ANALYSIS OF DURATIONS

fidelity to the time-out procedure (range $90-100 \%$ ).

Two independent observers trained to $90 \%$ interobserver agreement (IOA). One observer tracked sibling aggression in-vivo for all sessions using 5s partial interval recording with the aid of a headphone-delivered audio recording. A GoPro Hero3 camera recorded the sessions. After the sessions a different independent observer coded 33\% of videos for frequency of aggression and frequency and types of prompts used by the graduate assistant during the intervention. The videos were edited so that what happened immediately after instances of aggression was not included, insuring that the coder was blind to the treatment conditions. Percent agreement was determined by calculating the number of interval agreements between the in-vivo and coding observer divided by the total number of agreements plus disagreements, multiplied by 100 . Due to video timing issues, agreements were counted as long as the instance was recorded in the interval preceding or following the target instance. Intervals where one observer marked both children as aggressing and the other marked one child as aggressing counted as half an agreement. IOA remained high across all participants $(M=94.1$, range $83.3 \%$ to $100 \%)$.

The purpose of tracking prompts was to assess whether the graduate assistant, who was not blind to the conditions, differentially prompted to evoke less sibling aggression during timeout sessions than in the other sessions. The blind observer categorized prompts as general encouragement of play, encouragement to play with the same toy, or nonverbal placement of toy (e.g. "check this out," "go show your sister," and placing a toy between the two children, respectively). Prompt rates were fairly similar in baseline and time-out sessions, varying a minimum of .27 prompts per minute and a maximum of 1.47 prompts per minute. Encouraging play and encouraging play with the same toy were the two most common prompts. See Table 1 for prompt rates per condition and prompt-type percentages. 
TIME-OUT ANALYSIS OF DURATIONS

\section{Results}

\section{Across Subject Comparison}

Sibling aggression decreased to two instances or less per session for all participants with the use of a 1-min time-out contingent on every instance of aggression. Six participants had a clear reversal of behavior and two had a moderate reversal. The data show no differences in the effectiveness of a 1-min time-out between the older and younger children. A 1-min time-out was sufficient for all participants, thus we did not assess durations longer than 1-min. Parent-tracked follow-up data generally demonstrated maintenance of low levels of aggression one-week after the intervention. See Figure 1 for intervention and parent-tracked data for each participant.

\section{Lisa and Maddie ( 7 and 3 years old)}

Lisa and Maddie aggressed toward one another an average of 2.6 times per day over the two-week parent tracked baseline (range 0 to 8 ). During the in-home intervention baseline frequencies were above five instances per session for both Lisa and Maddie. Maddie exhibited extremely high frequencies of aggression during baseline sessions $(M=12.5)$. The first treatment session began with a 1-min time-out. Lisa and Maddie each received one 1-min timeout for aggressing and engaged in no further aggression during the first treatment session. Lisa's aggression returned to baseline levels during the return to baseline while Maddie's aggression increased but stayed below five instances per session $(M=3.5)$. The return to 1-min time-out treatment took place in two sessions. During the first session Lisa had five instances of aggression and decreased her aggression to zero instances during the second session. Maddie had one and zero instances of aggression during each session, respectively. Aggression during parent-collected follow-up maintained at low levels $(M=1.43)$ except for one day where there were seven instances of aggression. 
TIME-OUT ANALYSIS OF DURATIONS

\section{Tia and Tenley ( 4 and 3 years old)}

Tia and Tenley aggressed an average of eight times per day during the parent-tracked baseline. In-home intervention baseline frequencies were well above the inclusion criterion of five instances per minute ( $M=8.3$ for Tia, $M=10$ for Tenley). Tia and Tenley did not aggress during the first 1-min time-out treatment session; in the second session Tia aggressed once and Tenley aggressed twice. Aggression remained low during the return to baseline during the first session and increased substantially during the second baseline session (21 instances for both participants). The last 1-min time-out session resulted in only one instance of aggression for each participant. Levels of aggression remained at consistent, low levels during the parenttracked follow-up $(M=2.29)$.

\section{Maria and Ashley (5 and 3 years old)}

Maria and Ashley averaged 3.14 instances of aggression per day during the parenttracked baseline. Maria aggressed an average of 5 times and Ashley aggressed an average of 7.5 times during the in-home baseline. Three 1-min time-out sessions took place before the return to baseline in order to give Maria and Ashley enough opportunities to contact the time-out contingency. Maria and Ashley's aggression decreased to two instances each during the third time-out session. The return to baseline resulted in higher levels of aggression than in the initial baseline session for both participants. Upon return to the 1-min time-out treatment session, aggression decreased to three instances for Ashley and one instance for Maria during the first session and zero instances for both participants during the final session. Parent-tracked follow up revealed low levels of aggression $(M=1.6)$, with two days during which no aggression occurred.

\section{Dakota and Effie (4 and 3 years old)}


TIME-OUT ANALYSIS OF DURATIONS

During the parent-tracked baseline Dakota and Effie aggressed an average of 20 times per day. Baseline frequencies of aggression averaged 6 instances per minute for Dakota and 5.7 instances per minute for Effie. Aggression was lowest for both children during the second 1-min time-out session, dropping below the 2 instances per minute reversal criterion. Aggression increased slightly during the return to baseline ( $M=4.5$ for Dakota and $M=3.5$ for Effie) and decreased slightly during the return to 1 -min time-out $(M=3.5$ for Dakota and $M=2$ for Effie). Parent-tracked follow up was not a reliable source of maintenance data because the parent failed to consistently implement time-out and stopped using time-out altogether after a few days of inconsistent use.

\section{Discussion}

The current data suggest that a 1-min time-out was sufficient for reducing sibling aggression in 3-, 4-, 5-, and 7-year-old children, with these effects replicating for 3-and 4-yearolds in our study. Our sample included individuals with brain damage, developmental delays, and ADHD, suggesting that a 1-min time-out may be effective for neurodiverse individuals. A clear reversal of treatment effects for 6 of 8 participants and a moderate reversal for two participants (one with brain damage and ADHD diagnosis) suggest experimental control and behavior reduction resulting from time-out. Replication across and within ages provides preliminary support for a single, short duration of time-out for sibling aggression with children of various ages. An all-female demographic is distinct from past behavioral intervention research, in which the treatment population is often primarily male (Benjamin et al., 1983; Burchard \& Barrera, 1972; Donaldson \& Vollmer, 2012; Fabiano et al., 2004; Kapalka \& Bryk, 2007); replication of the current treatment effects across gender is recommended. Parent-tracked follow up data revealed short-term maintenance for all participants, with all follow-up aggression 
TIME-OUT ANALYSIS OF DURATIONS

frequencies below baseline levels. There was one outlier data point for Lisa and Maddie on the second day of follow up. Dakota and Effie's mother was not consistent with implementation of time-out post-training and therefore her follow-up data cannot be considered a reliable demonstration of the maintenance of time-out. Although there was a decrease in sibling aggression during follow-up, this may have been due to one of the children being sick.

The current study's design is unique in that it proposed an incremental increase assessment of time-out duration. Because no child required an increase to a 2-min time-out, the procedure was not fully carried out. On the other hand, this design eliminated the need for testing unnecessary durations. Past research has tested a handful of durations, comparing "short" and "long" time-outs, however the authors give no true rationale for why they select specific durations (Burchard \& Barrera, 1972; Hobbs et al., 1978; McGuffin, 1991; White et al., 1972). There are multiple benefits for using an incrementally increasing methodology. Primarily, the current methodology allowed for assessment of all minute-based durations until an effective duration was identified while avoiding sequence effects (Burchard \& Barrera, 1972; Kendall et al., 1975; White et al., 1972).

Demonstrating the effectiveness of a 1-min time-out for all participants in this study provides initial evidence to inform recommendations for best practice of time-out to parents. While the majority of parenting books and websites recommend the 1-min per year of age rule (Clark, 2005; Frost, 2014; Reece, 2013; Smith \& Chandler, 2010; Zolten \& Long, 1997), current results and past research question the legitimacy of this rule (Kapalka \& Bryk, 2007). A 1-min time-out provides several important advantages, such as requiring less monitoring, being less time consuming, and being less intrusive than a longer time-out. Parents with children who frequently exhibit problem behavior while in time-out may also find shorter durations easier to 
TIME-OUT ANALYSIS OF DURATIONS

manage than longer durations. A short duration of time-out (e.g. 1-min) also allows for multiple learning trials of the consequences for inappropriate behavior to occur in a short amount of time; this facilitates an optimal balance of time spent for behavior reduction and time spent for behavior acquisition. Parents were pleased with the intervention, with all parents reporting being either somewhat likely or extremely likely to recommend the training to someone else and use the intervention in the future, and reporting good overall satisfaction with the training they received.

While the current findings are encouraging in terms of a short duration time-out (e.g., 1min), several limitations suggests the need for further research. First, it is possible that our results may be specific to low levels of sibling aggression for short periods of time with a monitoring adult. Further research should examine a similar methodology with other problem behaviors. Another limitation was the presence of a graduate assistant encouraging play between siblings and at times contriving opportunities where aggression could occur. Contrived play was used in order to condense a less frequent behavior (sibling aggression) into a time-frame where baseline and intervention sessions could occur while we were present to directly observe behavior. This could have resulted in interactions that would not typically occur in the absence of the research assistant, although anecdotally one parent said the types of situations being contrived were very similar to everyday situations. Because the graduate assistant was not blind to condition, differential prompting of aggressive behaviors could have occurred. To test for this possibility, a blind observer watched segments of time-out and baseline sessions for each participant and counted the frequency of different types of prompts. The blind observer identified similar rates of prompting in time-out and baseline sessions for all participants. The type and rate of prompting used was specific to each set of participants—some children needed 
TIME-OUT ANALYSIS OF DURATIONS

more prompting than others to maintain continuous joint play. Therefore, higher rates of prompting during certain sessions may be indicative of decreased willingness of the participants to play together rather than differential prompting by the graduate assistant.

Another limitation was our presence during all sessions of the intervention. We could have become a discriminative stimulus (SD) for time-out; in other words, we could have become associated with time-out, such that the target behavior would only result in time-out when we were present. Thus, when we were absent (the S-delta for punishment by time-out), the newly learned behavior associated with avoidance of time-out did not generalize because we (the SD for time-out) were not present. One attempt to increase generalization of results across time was to conduct the intervention in the participants' homes with the parent. The downside of a naturalistic setting is loss of control. Participants were able to leave the room without much difficulty, and distractions such as visitors and non-relevant toys were present. Another limitation is the lack of long-term maintenance data. Although parent-tracked follow up suggests that treatment effects maintain for 1-week following the intervention, the effectiveness of a 1min time-out could fade after multiple months.

Generalization of our findings may be limited due to certain characteristics of our participants. Because our participants were all female, we cannot make statements about the efficacy of 1-min time-outs for male children. Three of the eight participants were diagnosed with static encephalopathy, Noonan Syndrome, and/or ADHD, thus limiting the generalizability of results to a typically developing population. However, five of the eight participants were typically developing and all five showed a reduction in sibling aggression with a 1-minute timeout, which suggests that the results may still be generalizable. Additionally, we did not control for age difference in sibling dyads (differences ranged from one to four years). The reduction in 
TIME-OUT ANALYSIS OF DURATIONS

sibling aggression across all dyads suggests that 1-min time-outs may be generalizable to sibling dyads with age differences of one, two, and four years.

Future research should address the issue of the long-term effectiveness of a 1-min timeout as well as seek to replicate the current findings within and across populations and problem behaviors. Because the current study was the first of its kind and only included siblings between 3 and 7 years old, future research should seek to replicate results with the same population. With replication a slight modification to methodology should be considered: setting a minimum number of sessions per condition. This would help to standardize and remove bias from condition changes. Future investigators may also consider conducting sessions across multiple days to account for day-to-day variability in child behavior. Past duration research has not used the current incremental duration increase method, suggesting that further duration research with individuals diagnosed with developmental disabilities may be beneficial. Finally, research should be conducted on how to best disseminate evidence-based parenting recommendations. 


\section{References}

Adams, C. D., \& Kelley, M. L. (1992). Managing sibling aggression: Overcorrection as an alternative to time-out. Behavior Therapy, 23, 707-717. doi:10.1016/S00057894(05)80230-8

Allison, T. S., \& Allison, S. L. (1971). Time-out from reinforcement: Effect on sibling aggression. The Psychological Record, 21, 81-86. Retrieved from http://thepsychologicalrecord.siu.edu

Baer, D. M. (1961). Effect of withdrawal of positive reinforcement on an extinguishing response in young children. Child Development, 32, 67-74. doi:10.1111/j.14678624.1961.tb05004.x

Barton, L. E., Brulle, A. R., \& Repp, A. C. (1987). Effects of differential scheduling of timeout to reduce maladaptive responding. Exceptional Children, 53, 351-356. Retrieved from http://journals.sagepub.com/doi/pdf/10.1177/001440298705300410

Benjamin, R., Mazzarins, H., \& Kupfersmid, J. (1983). The effect of time-out (TO) duration on assaultiveness in psychiatrically hospitalized children. Aggressive Behavior, 9, 21-27. doi:10.1002/1098-2337(1983)9:13.0.CO;2-H

Bostow, D. E., \& Bailey, J. B. (1969). Modification of severe disruptive and aggressive behavior using brief timeout and reinforcement procedures. Journal of Applied Behavior Analysis, 2, 31-37. doi:10.1901/jaba.1969.2-31

Burchard, J. D., \& Barrera, F. (1972). An analysis of timeout and response cost in a programmed 
TIME-OUT ANALYSIS OF DURATIONS

environment. Journal of Applied Behavior Analysis, 5, 271-282.

doi:10.1901/jaba.1972.5-271

Clark, L. (2005). SOS: Help for parents (3rd ed.). Bowling Green, KY: SOS Programs \& Parents Press

Donaldson, J. M., \& Vollmer, T. R. (2011). An evaluation and comparison of time-out procedures with and without release contingencies. Journal of Applied Behavior Analysis, 44, 693-705. doi:10.1901/jaba.2011.44-693

Donaldson, J. M., \& Vollmer, T. R. (2012). A procedure for thinning the schedule of time-out. Journal of Applied Behavior Analysis, 45, 625-630. doi:10.1901/jaba.2012.45-625

Dunn, J. (1983). Sibling relationships in early childhood. Child Development, 54, 787-811. http://dx.doi.org.pacificlib.idm.oclc.org/10.2307/1129886

Fabiano, G. A., Pelham Jr., W. E., Manos, M. J., Gnagy, M. E., Chronis, A. M., Onyango, A. N., ... Swain, S. (2004). An evaluation of three time-out procedures for children with attention-deficit/hyperactivity disorder. Behavior Therapy, 35, 449-469. doi:10.1016/S0005-7894(04)80027-3

Frost, J. (2014). Jo Frost's toddler rules: Your 5-step guide to shaping proper behavior. New York: Ballantine Books.

Hobbs, S. A., Forehand, R., \& Murray, R. G. (1978). Effects of various durations of timeout on the noncompliant behavior of children. Behavior Therapy, 9, 652-656. doi:10.1016/S0005-7894(78)80142-7

James, J. E. (1976). The influence of duration on the effects of time-out from speaking. Journal of Speech, Language, and Hearing Research, 19, 206-215. doi:10.1044/jshr.1902.206

Jensen, S. A., Blumberg, S., \& Doerr, M. (2017). Structured feedback training for time out: 
TIME-OUT ANALYSIS OF DURATIONS

Efficacy and efficiency in comparison to a didactic method. Manuscript submitted for publication.

Jones, R. N., Sloane, H. N., \& Roberts, M. W. (1992). Limitations of “don't” instructional control. Behavior Therapy, 23, 131-140. http://dx.doi.org.pacificlib.idm.oclc.org/10.1016/S0005-7894(05)80313-2

Kapalka, G. M., \& Bryk, L. J. (2007). Two- to four- minute time-out is sufficient for young boys with ADHD. Early Childhood Services, 1, 181-188. Retrieved from https://www.pluralpublishing.com/journals_ECS.htm

Kendall, P. C., Nay, W. R., \& Jeffers, J. (1975). Timeout duration and contrast effects: A systematic evaluation of a successive treatments design. Behavior Therapy, 6, 609-615. doi:10.1016/S0005-7894(75)80182-1

Kramer, L., \& Baron, L. A. (1995). Parental perceptions of children's sibling relationships. Family Relations, 44, 95-103. http://dx.doi.org.pacificlib.idm.oclc.org/10.2307/584746

Leitenberg, H. (1965). Is time-out from positive reinforcement an aversive event? A review of the experimental evidence. Psychological Bulletin, 64, 428-441. http://dx.doi.org.pacificlib.idm.oclc.org/10.1037/h0022657

Marcus, B. A., Swanson, V., \& Vollmer, T. R. (2001). Effects of parent training on parent and child behavior using procedures based on functional analyses. Behavioral Interventions, 16, 87-104. doi:10.1002/bin.87

MacDonough, T. S., \& Forehand, R. (1973). Response-contingent time out: Important parameters in behavior modification with children. Journal of Behavior Therapy and Experimental Psychiatry, 4, 231-236.

http://dx.doi.org.pacificlib.idm.oclc.org/10.1016/0005-7916(73)90079-7 
TIME-OUT ANALYSIS OF DURATIONS

McGuffin, P. W. (1991). The effect of timeout duration on frequency of aggression in hospitalized children with conduct disorders. Behavioral Residential Treatment, 6, 279288. doi:10.1002/bin.2360060405

O’Leary, K. D., O’Leary, S., \& Becker, W. C. (1967). Modification of a deviant sibling interaction pattern in the home. Behavior Research and Therapy, 5, 113-120. http://dx.doi.org.pacificlib.idm.oclc.org/10.1016/0005-7967(67)90005-8

Olson, R. L., \& Roberts, M. W. (1987). Alternative treatments for sibling aggression. Behavior Therapy, 18, 243-250. http://dx.doi.org.pacificlib.idm.oclc.org/10.1016/S00057894(87)80018-7

Pendergrass, V. E. (1971). Effects of length of time-out from positive reinforcement and schedule of application in suppression of aggressive behavior. The Psychological Record, 21, 75-80. Retrieved from http://thepsychologicalrecord.siu.edu

Reece, T. (2013). 6 successful time-out tactics. Retrieved from http://www.parents.com/toddlerspreschoolers/discipline/time-out/successful-time-out-tactics/

Smith, M., \& Chandler, R. (2010). Toddler ABC guide to discipline: Quick secrets to loving guidance. United States: Author.

Straus, M. A., Gelles, R. J., \& Steinmetz, S. K. (1980). Behind closed doors: Violence in the American family. Garden City, NY: Doubleday.

Wahler, R. G. (1969). Oppositional children: A quest for parental reinforcement control. Journal of Applied Behavior Analysis, 2, 159-170. http://dx.doi.org.pacificlib.idm.oclc.org/10.1901/jaba.1969.2-159

White, G. D., Nielsen, G., \& Johnson, S. M. (1972). Timeout duration and the suppression of deviant behavior in children. Journal of Applied Behavior Analysis, 5, 111-120. 
TIME-OUT ANALYSIS OF DURATIONS

http://dx.doi.org.pacificlib.idm.oclc.org/10.1901/jaba.1972.5-111

Zolten, K., \& Long, N. Time-out as a discipline technique. Retrieved from http://www.parentinged.org/handouts/timeout.pdf 